\title{
Aerobic and Anaerobic Biotransformation of Bile Acids by Escherichia coli (I)
}

\author{
Yoshio Ogura, ${ }^{1 *}$ Nobuo Yamaga, ${ }^{1}$ Yasuhiro Kido, ${ }^{2}$ Rie Katayama, ${ }^{1}$ Kazuo Yamada ${ }^{1}$ and \\ Kiyohisa UCHIDA ${ }^{1}$ \\ 'Division of Medical Biochemistry, Department of Pathophysiological and Therapeutic Science, School of Medicine, \\ Tottori University, Yonago 683-8503, Japan \\ ${ }^{2}$ Department of Food Sciences and Nutritional Health, Faculty of Human Environment, Kyoto Prefectural University, Kyoto 606-8522, \\ Japan
}

Received March 7, 2003; Accepted for publication, September 1, 2003

The effects of Escherichia coli on the transformation of bile acids were examined in aerobic and anaerobic culturing systems for 4 days. Bile acids were converted to methyl ester dimethylethylsilyl ether derivatives and determined by capillary gas liquid chromatography. $E$. coli oxidized cholic acid (CA) and chenodeoxycholic acid (CDCA) to $3 \alpha, 12 \alpha-$ dihydroxy-7-oxo-5 $\beta$-cholanoic acid $(3 \alpha 12 \alpha 7=0)$ and $3 \alpha$-hydroxy-7-oxo-5 $\beta$-cholanoic acid $(3 \alpha 7=0)$, and reduced these oxo-bile acids to CA and CDCA, respectively. These oxidation and reduction reactions occurred in both aerobic and anaerobic cultures, finally attaining certain equilibrium values. The values, or oxidation/reduction ratios, were 9 or more in the aerobic culture, and about 1 in the anaerobic culture. $E$. coli also oxidized taurocholic acid in the aerobic culture, but not in the anaerobic culture.

Key words: Escherichia coli; cholic acid; chenodeoxycholic acid; taurocholic acid; in vitro biotransformation

\section{INTRODUCTION}

Escherichia coli possesses $7 \alpha$-hydroxysteroid dehydrogenase $(7 \alpha-\mathrm{HSDH})(2,12) .7 \alpha-\mathrm{HSDH}$ is a non-inducible intracellular enzyme of substrate specificity for bile acids (both free and conjugated) having a $7 \alpha$-hydroxyl group $(7,12)$ and requiring $\mathrm{NAD}^{+}$as a cofactor for the activity $(12,15)$. Therefore, $7 \alpha$-hydroxyl bile acids are oxidized to the corresponding 7-oxo-bile acids when incubated with $E$. coli in vitro $(1,9,13)$. However, no detectable tranformation of endogenous bile acids was observed in gnotobiote rats inoculated with E. $\operatorname{coli}(4,10,17)$.

We speculated that this discrepancy between in vitro and in vivo experiments was due to the presence or absence of oxygen, and so we examined the activity of $E$. coli on bile acid transformation in both aerobic and anaerobic culturing systems.

\section{MATERIALS AND METHODS}

Chemicals. Cholic acid (CA), chenodeoxycholic acid (CDCA), and taurocholic acid (TCA) were purchased from Sigma Chemicals Co., Missouri, USA. $3 \alpha$, $12 \alpha$-Dihydroxy-7-oxo- $5 \beta$-cholanoic acid $(3 \alpha 12 \alpha 7=0)$

\footnotetext{
*Corresponding author. Mailing address: Division of Medical Biochemis try, Department of Pathophysiological and Therapeutic Science, School of Medicine, Tottori University, 86, Nishi-cho, Yonago 683-8503, Japan. Phone: +81-859-34-8010. Fax: +81-859-34-8077. E-mail: ogura@grape.med. tottori-u.ac.jp
}

and $3 \alpha$-hydroxy-7-oxo- $5 \beta$-cholanoic acid ( $3 \alpha 7=0)$ were prepared by the oxidation of CA and CDCA with $N$-bromosuccinimide (5), respectively. $7 \beta, 12 \beta$ Dihydroxy- $5 \beta$-cholanoic acid $(\beta \beta)$, an internal standard for analysis by capillary gas liquid chromatography (GLC), was synthesized as previously described (3). These bile acids were estimated to be more than $96 \%$ pure by capillary GLC. Piperidino hydroxy propyl dextran gel (PHP GEL) was purchased from Shimadzu, Kyoto, Japan, and dimethylethylsilylimidazole from Tokyo Kasei Kogyo Co. Ltd., Tokyo, Japan. The other reagents and solvents of analytical grade were obtained from Wako Pure Chemical Industries, Osaka, Japan. If not otherwise stated, the solvents were distilled once before use.

Bacteriological procedures. Two strains of $E$. coli were used. E. coli K-12 was obtained from the American Type Culture Collection, Virginia, USA, and $E$. coli HB101 was obtained from Takara Shuzo Co. Ltd., Shiga, Japan. Brain-heart infusion (BHI: Becton Dickinson, Maryland, USA) was used in all tests.

$E$. coli was precultured aerobically in the BHI medium at $37^{\circ} \mathrm{C}$ for 1 day. Aliquots $\left(3 \times 10^{7} / 10 \mu \mathrm{l}\right)$ of $E$. coli were added to $2 \mathrm{ml}$ of $\mathrm{BHI}$ medium containing 1 $\mathrm{mmol} / \mathrm{l}$ of bile acids and cultured at $37^{\circ} \mathrm{C}$ for 4 days. Aerobic culturing was performed by standing in a thermostat and anaerobic culturing was performed using the gas-pack method (BBL ${ }^{\mathrm{TM}}$ Gas Pak Pouch ${ }^{\mathrm{TM}}$ System; Becton Dickinson, Maryland, USA). 
Analytical methods. A portion of the medium cultured, $100 \mu \mathrm{l}$, was treated with 8 volumes of ethanol by warming for $10 \mathrm{~min}$ and then filtered. The filtrate was evaporated to dryness under a stream of nitrogen and the residue was hydrolyzed in alkaline solution (20). After hydrolysis, the reaction mixture was acidified with diluted hydrochloric acid and bile acids were extracted with ethylether. The extracted bile acids were converted into methyl ester dimethylethylsilyl ether (Me-DMES) derivatives as described previously $(18,19)$. Briefly, bile acids were methylated with diazomethane, and then left in $70 \mu \mathrm{l}$ of dimethylethylsilylimidazole in an airtight vessel at room temperature overnight.

In the case of TCA, bile acids in the medium were separated to free, glycine-conjugate and taurineconjugate fractions using a PHP GEL column (18). Glycine- and taurine-conjugate fractions were hydrolyzed in alkali and analyzed by GLC as described above.

$3 \alpha$-Hydroxy-7-oxo-5 $\beta$-cholanoic acid methyl ester was converted to its methoxime derivative as described by Horning et al. (8), and then to a DMES derivative to separately determine this compound and CDCA by GLC.

Capillary gas liquid chromatography. An aliquot of the bile acid derivatives dissolved in $n$-hexane was injected into a gas chromatograph (Model GC-14A, Shimadzu, Kyoto, Japan) equipped with a flame ionization detector, a solventless injector, and a comput-

Table 1. Relative retention times of bile acid derivatives.

\begin{tabular}{lcc}
\multicolumn{1}{c}{ Derivatives } & $\begin{array}{c}\text { Retention time } \\
(\mathrm{min})\end{array}$ & $\begin{array}{c}\text { Relative retention } \\
\text { time }\end{array}$ \\
\hline $7 \beta, 12 \beta$-Dihydroxy-5 $\beta$-cholanoic acid Me-DMES & $12.57^{\mathrm{a})}$ & 1.00 \\
Chenodeoxycholic acid Me-DMES & & 1.16 \\
$3 \alpha$-Hydroxy-7-oxo-5 $\beta$-cholanoic acid Me-DMES & & 1.16 \\
$3 \alpha$-Hydroxy-7-oxo-5 $\beta$-cholanoic acid Me-MO-DMES & & 1.05 \\
Cholic acid Me-DMES & & 1.47 \\
$3 \alpha, 12 \alpha$-Dihydroxy-7-oxo-5 $\beta$-cholanoic acid Me-DMES & & 1.57 \\
Unknown & & 1.76 \\
\hline
\end{tabular}

a) This value varies slightly depending on individual experiments.

Me-DMES: Methyl ester dimethylethylsilyl ether.

Me-MO-DMES: Methyl ester methoxime dimethylethylsilyl ether.

A) $\mathrm{CA}$ (aerobic)

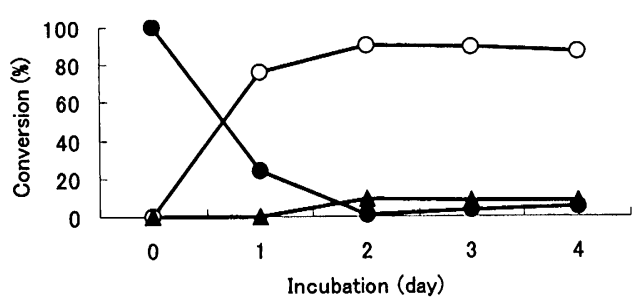

C) $\mathrm{CA}$ (anaerobic)

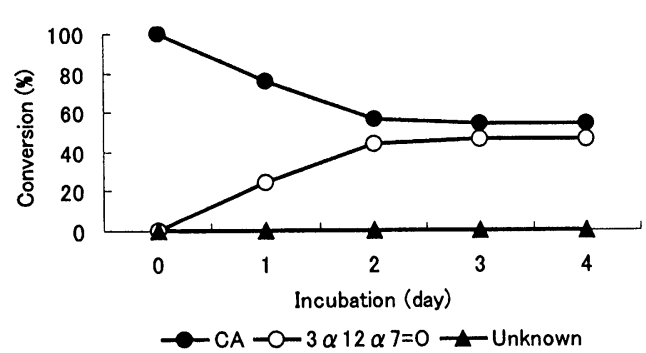

B) $3 \alpha 12 \alpha 7=0$ (aerobic)

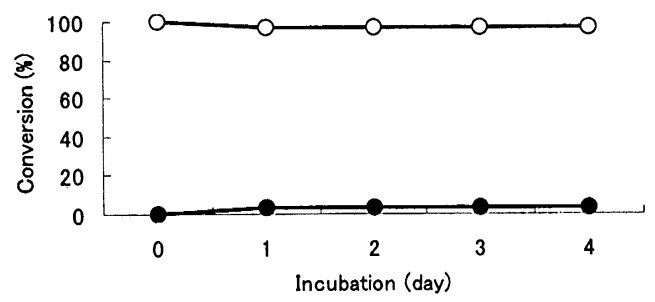

D) $3 \alpha 12 \alpha 7=0$ (anaerobic)

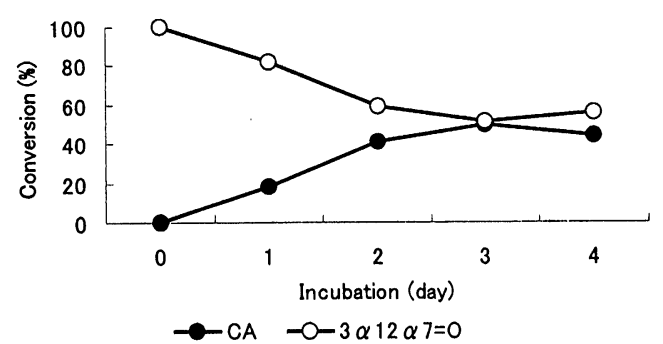

Fig. 1. Cholic acid (CA) and $3 \alpha, 12 \alpha$-dihydroxy-7-oxo-5 $\beta$-cholanoic acid ( $3 \alpha 12 \alpha 7=0)$ conversions by Escherichia coli $\mathrm{K}-12$. 

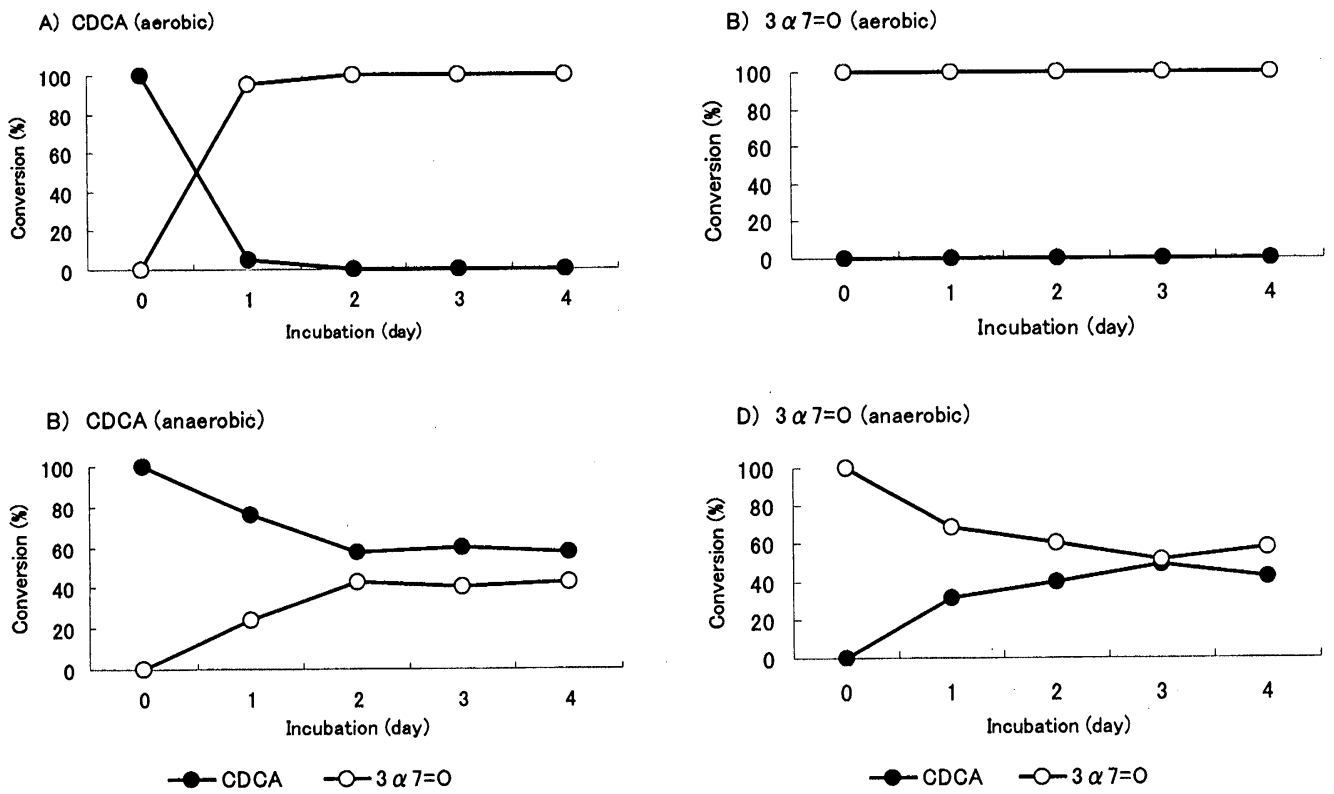

Fig. 2. Chenodeoxycholic acid (CDCA) and $3 \alpha$-hydroxy-7-oxo-5 $\beta$-cholanoic acid ( $3 \alpha 7=0)$ conversions by Escherichia coli K-12.

erized data system (Model C-R4A, Shimadzu, Kyoto, Japan). A Hicap CBP-1 capillary column $(25 \mathrm{~m} \times 0.25$ $\mathrm{mm}$ i.d., Shimadzu, Kyoto, Japan) was used. The column temperature was maintained at $285^{\circ} \mathrm{C}$ and helium was used as the carrier gas.

Relative retention times of bile acid derivatives obtained during the GLC analysis are given in Table 1.

\section{RESULTS}

The time course of changes in the $7 \alpha$-dehydrogenation of CA and $7 \alpha$-hydrogenation of $3 \alpha 12 \alpha 7=\mathrm{O}$ by $E$. coli $\mathrm{K}-12$ are shown in Fig. 1. In the aerobic culture, CA was rapidly oxidized to $3 \alpha 12 \alpha 7=0$, about $80 \%$ in 1 day and almost to a maximum in 2 days. Small amounts of unidentified metabolites were detected after 2 days. On the other hand, the reverse reaction, reduction of $3 \alpha 12 \alpha 7=\mathrm{O}$ to $\mathrm{CA}$, was minor in this system. In the anaerobic culture, the oxidation of CA progressed, but more slowly than in the aerobic culture, and attained a constant value (about 50\%) after 2 days. The reduction of $3 \alpha 12 \alpha 7=0$ also progressed in a similar manner as the oxidation of CA and attained a constant value after 2 days. The activity of another strain of E. coli, $\mathrm{HB} 101$, on CA conversion in the aerobic culture was examined, and it was found the activity was similar to that of $\mathrm{K}-12$ (data are not given).

Figure 2 shows the time course of changes in the $7 \alpha-$ dehydrogenation of CDCA and the reverse reaction by E. coli $\mathrm{K}-12$. The changes are very similar to those in

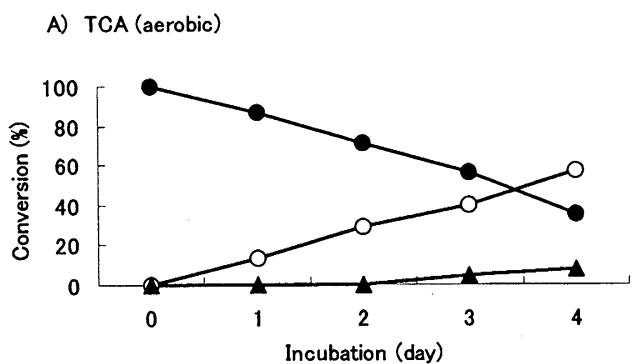

B) TCA (anaerobic)

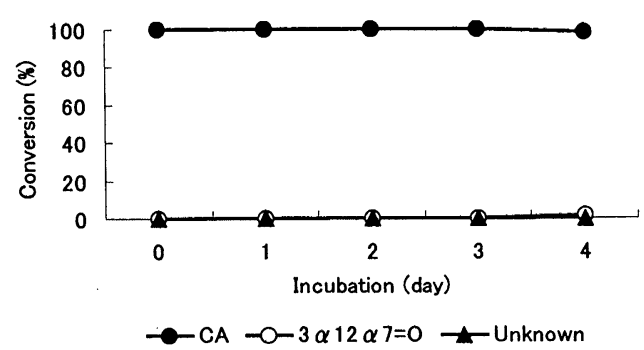

Fig. 3. Taurocholic acid (TCA) conversion by Escherichia coli $\mathrm{K}-12$.

CA and $3 \alpha 12 \alpha 7=0$, but the oxidation of CDCA in the aerobic culture seemed to proceed more rapidly than that of CA.

E. coli also dehydrogenated TCA. This reaction was observed in the aerobic culture, but not in the anaerobic culture (Fig. 3). The aerobic dehydrogenation of 

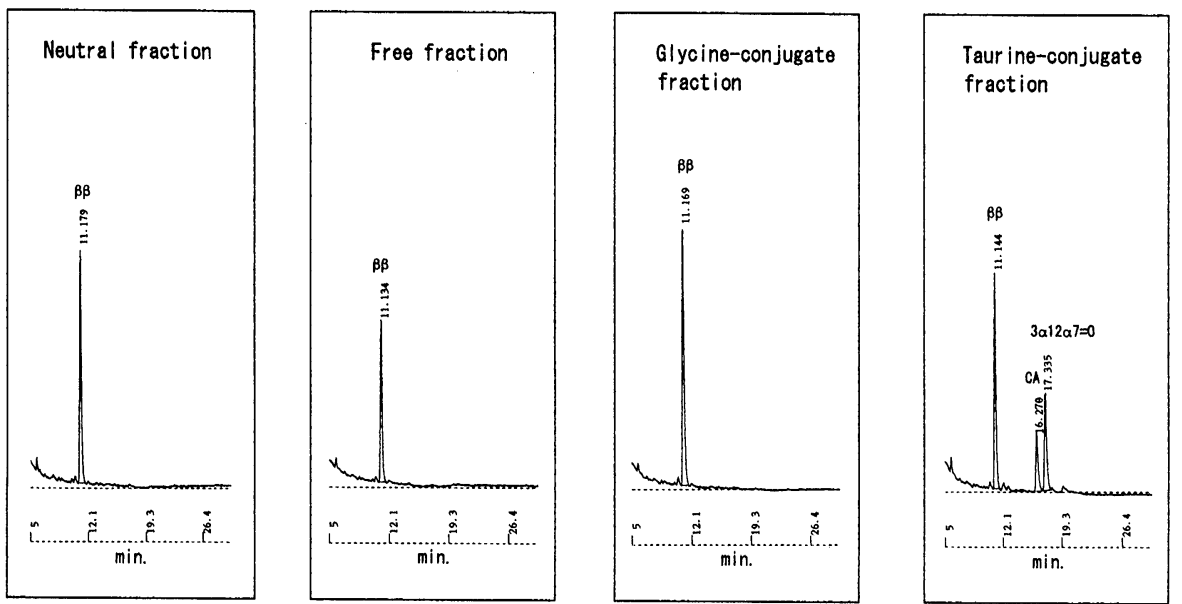

Fig. 4. Gas-chromatograms of neutral, free, glycine- and taurine-conjugated fractions by PHP GEL column from the cultured medium containing taurocholic acid (TCA) for 4 days.

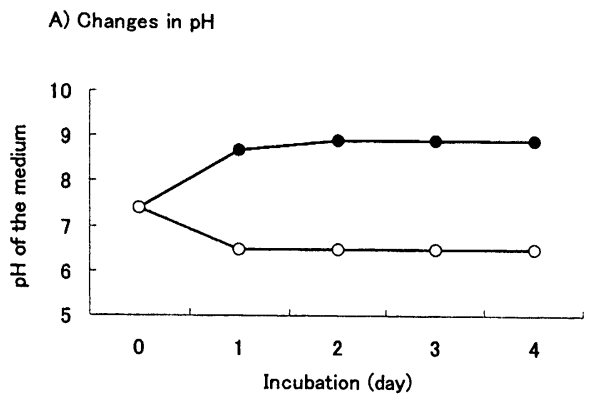

B) Viable E. coli

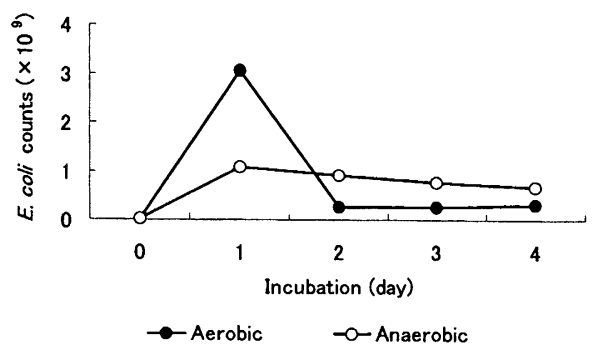

Fig. 5. Changes in $\mathrm{pH}$ of the medium and viable $E$. coli counts during culturing with Escherichia coli K-12.

TCA occurred more slowly than CA.

In the case of TCA, the 7-oxo-bile acid formed was found only in the taurine-conjugate fraction (Fig. 4). This suggests that TCA was oxidized without deconjugation. No bile acids, including CA, were detected in the free-fraction, suggesting that $E$. coli has no activity of deconjugation.

Figure 5 shows changes in the $\mathrm{pH}$ of the medium with $E$. coli and in viable $E$. coli counts during aerobic and anaerobic culturing. The $\mathrm{pH}$ increased to about 9 in the aerobic culture, but decreased slightly to around 6.5 in the anaerobic culture from day 1 , and these values remained the same thereafter (Fig. 5A). The viable $E$. coli counts increased about $3 \times 10^{9} / \mathrm{ml}$ in the aerobic culture on day 1 , but decreased remarkably after the second day. In the anaerobic culture, it increased about $1 \times 10^{9} / \mathrm{ml}$ on day 1 , and then decreased gradually. As for the viable $E$. coli count, there was more in the anaerobic culture than in the aerobic culture after the second day (Fig. 5B). These changes were reproducible in all the other experiments.

\section{DISCUSSION}

E. coli possesses $7 \alpha-\operatorname{HSDH}(2,12)$ and effectively oxidizes CA and CDCA to the corresponding 7-oxobile acids when incubated in vitro $(9,13,14,16)$, while E. coli exhibits only minor activity on endogenous bile acids in gnotobiotic rats $(4,10)$.

So far, as we have examined in the present culturing systems, E. coli oxidized CA and CDCA to $3 \alpha 12 \alpha$ $7=\mathrm{O}$ and $3 \alpha 7=\mathrm{O}$, and reduced these oxo-bile acids to CA and CDCA, respectively. These oxidation and reduction reactions seem to attain different equilibrium values depending on the presence or absence of oxygen. When the equilibrium value was expressed by an oxidation/reduction $(\mathrm{O} / \mathrm{R})$ ratio, it was 9 or more under aerobic conditions and around 1 under anaerobic conditions for both CA and CDCA. The equilibrium was attained in about 2 days.

How oxygen affects the equilibrium value is not yet known, but the reaction of this enzyme is reported to be dependent on the $\mathrm{pH}$ of the medium. Oxidation is predominant in values above 7 as reduction is in values 
below $7(6,11)$. In the present experiments, the $\mathrm{pH}$ of the medium increased to about 9 in the aerobic culture but decreased to about 6.5 in the anaerobic culture. These changes are consistent with changes in the equilibrium values $(\mathrm{O} / \mathrm{R}$ ratios).

It is reported that the enzyme has about one order of magnitude higher affinity for CDCA than for CA (12, 15). In the present experiments, the oxidation of CDCA was faster than that of $\mathrm{CA}$ in the aerobic culture, but was almost the same in the anaerobic culture.

TCA was also oxidized to the corresponding taurineconjugated 7-oxo-bile acid. However, this reaction occurred only in the aerobic cultures, and not in the anaerobic culture (Fig. 3). Aries and Hill (2) and Imamura et al. (9) also noted that TCA was hardly dehydrogenated by $E$. coli under anaerobic conditions.

These data explain the fact that $E$. coli possesses $7 \alpha$ HSDH but causes almost no effect on the transformation of bile acids in vivo. Bile acids in germ-free rats consist of mainly taurine-conjugated primary bile acids such as CA, CDCA and $\alpha$ - and $\beta$-muricholic acids (10). Therefore, taurine-conjugated $7 \alpha$-hydroxyl bile acids are rarely oxidized in $E$. coli inoculated gnotobiote rats $(4,10)$.

An anaerobic condition is considered to be one of the reasons that the conversion of bile acids was not observed in vivo, but there are many factors that affect the biotransformation of bile acids in vivo. Further studies are necessary to clarify the exact mechanisms.

\section{REFERENCES}

(1) Aries V, Crowther JS, Drasar BS, Hill MJ. 1969. Degradation of bile salts by human intestinal bacteria. Gut 10: 575576.

(2) Aries V, Hill MJ. 1970. Degradation of steroids by intestinal bacteria. II. Enzymes catalyzing the oxidoreduction of the $3 \alpha-, 7 \alpha$-, and $12 \alpha$-hydroxyl groups in cholic acid, and the dehydroxylation of the 7-hydroxyl group. Biochim Biophys Acta 202: 535-543.

(3) Arimoto K, Adachi K, Yamaga N. 1982. $7 \beta, 12 \beta$ Dihydroxy-5 $\beta$-cholan-24-oic acid as an internal standard for quantitative determination of bile acids by gas chromatography. Steroids 39: 631-641.

(4) Chikai T, Nakao H, Uchida K. 1987. Deconjugation of bile acids by human intestinal bacteria implanted in germ-free rats. Lipids 22: 669-671.

(5) Fieser LF, Rajagopalan S. 1949. Selective oxidation with
$N$-bromosuccinimide. I. Cholic acid. J Am Chem Soc 71: 3935-3938.

(6) Groh H, Schade K, Hörhold-Schubert C. 1993. Steroid metabolism with intestinal microorganisms. J Basic Microbiol 33: $59-72$.

(7) Haslewood ES, Haslewood GAD. 1976. The specificity of a $7 \alpha$-hydroxysteroid dehydrogenase from Escherichia coli. Biochem J 157: 207-210.

(8) Horning GH, Moss MA, Horning CE. 1968. Formation and gas-liquid chromatographic behavior of isometric steroid ketone methoxime derivatives. Anal Biochem 22: 284-294.

(9) Imamura T, Sakamoto N, Tamaki M, Hirano S. 1979. Transformation of bile acids by members of Enterobacteriaceae. Jpn J Bacteriol 34: 513-520.

(10) Kayahara T, Tamura T, Amuro Y, Higashino K, Igimi H, Uchida K. 1994. $\Delta^{22}-\beta$-Muricholic acid in monoassociated rats and conventional rats. Lipids 29: 289-296.

(11) Macdonald IA, Bokkenheuser VD, Winter J, McLernon AM, Mosbach EH. 1983. Degradation of steroids in the human gut. J Lipid Res 24: 675-700.

(12) Macdonald IA, Williams CN, Mahony DE. 1973. $7 \alpha$ Hydroxysteroid dehydrogenase from Escherichia coli B: Preliminary studies. Biochim Biophys Acta 309: 243-253.

(13) Midtvedt T, Norman A. 1967. Bile acid transformations by microbial strains belonging to genera found in intestinal contents. Acta Pathol Microbiol Scand 71: 629-638.

(14) Norman A, Bergman S. 1960. The action of intestinal microorganisms on bile acids. Bile acids and steroids 101 . Acta Chem Scand 14: 1781-1789.

(15) Prabha V, Gupta M, Gupta KG. 1989. Kinetic properties of $7 \alpha$-hydroxysteroid dehydrogenase from Escherichia coli 080. Can J Microbiol 35: 1076-1080.

(16) Suzuki R. 1970. Influence of intestinal microorganisms on the metabolism of bile acids in mice. Keio J Med 19: 7386.

(17) Uchida K, Satoh T, Narushima S, Itoh K, Takase H, Kuruma K, Nakao H, Yamaga N, Yamada K. 1999. Transformation of bile acids and sterols by Clostridia (fusiform bacteria) in Wistar rats. Lipids 34: 269-273.

(18) Yamaga N, Adachi K, Kohara H, Shimizu K. 1987. Internal standards for quantitative gas chromatography of individual bile acids after group separation of bile acids in urine. J Chromatogr Biomed Appl 422: 25-32.

(19) Yamaga N, Ikebuchi J, Kohara H, Ogura Y, Yamada K. 1996. Analysis of bile acids in urine specimens from healthy humans: Determination of several bile acids with $\beta$-hydroxyl and carbonyl groups. J Biochem 119: 725-730.

(20) Yamaga N, Nagano Y, Yamada K. 1997. An examination of alkaline hydrolyzing conditions of conjugated bile acids with carbonyl groups. Yonago Acta Med 40: 73-77. 